\title{
Computer Graphics: New Horizons in the Education of Lighting Designers and Engineers*
}

\author{
Vladimir Budak ${ }^{1[0000-0003-4750-0160]}$, and Denis Makarov 20000-0002-5043-8911] \\ ${ }^{1}$ National research university "MPEI”, 111250 Moscow, Russia \\ budakvp@gmail.com \\ ${ }^{2}$ CJSC "Point of Support Promelektrosvet", 125080 Moscow, \\ Russia \\ d.makarov@k-to.ru
}

\begin{abstract}
The development of computer graphics programs (CGP) and their widespread introduction into the practice of designing indoor and outdoor lighting with the ability to visualize the projected object has led to the fact that the design engineer simultaneously performs the functions of a designer. Similarly, designers creating artistic light images that previously assumed the selection and adjustment of light on the spot are now forced to select real lighting devices using PKG, which requires professional engineering knowledge. It determines the objective basis for creating a new specialty - light designer. However, the systems of engineering and art education have almost no common methodological foundations. We propose a method of teaching based on the use of PCG in the educational process, which will be able to combine both educational systems into a single specialty. The methodology is based on the joint project created with the teacher in CGP, collective discussion, and defense of the developed projects. It allows engineers to explain the created artistic images, and designers to explain the influence of the characteristics of light fields and parameters of lighting devices on the image. The results of test sessions among students in both areas of study are discussed. The thinking features of each student group and the possibility of developing a unified approach to design are analyzed.
\end{abstract}

Keywords: Lighting design, Lighting engineering, 3D modeling, Lighting modeling, Education.

\section{Introduction}

Until recently, there were two different approaches to lighting design. In the first engineering approach, the design of lighting installation (LI) was carried out to create

Copyright c 2020 for this paper by its authors. Use permitted under Creative Commons License Attribution 4.0 International (CC BY 4.0).

* Publication supported by RFBR grant № 19-01-00435 
the necessary levels of illumination and quality of lighting (discomfort, ripples, color reproduction) for performing some work by a person. In the second design approach, the light artist with architectural or exposure lighting sought to create some image, believing that the light would already be adjusted in place.

The rapid development of both directions of computer graphics in design, which allowed how to create a virtual image of the projected object, in this case, perform a rigorous calculation of light distribution taking into account all the processes of reflection and transmission of light, rapidly drew together both strands and identified the emergence of a new profession - lighting design.

However, the birth of a new specialty did not lead to creating a unified training program for them. There were still two directions in education, in one of which engineering taught "the physics of light," and in the other - artistic "the feeling of light" [1]. In engineering education, the basis is studying the nature of the light field, calculating its parameters, and the psychophysiology of its perception by the human eye. In art education, the student is taught taste through drawing design objects and discussing it with a mentor - learning "on the tip of a pencil."

At the Department of lighting engineering of the National research university "MPEI," teaching students to perceive and feel light in the framework of engineering education has been dealt with for more than a decade. For example, in their work [2], the authors touch on the principles of similarity of nature and image sensations. A little later, in [3], it is proposed to structure modeling lighting in groups to find the identity of physical models with calculated visual sensations.

In art education, serious attempts have been made to link the "feeling of light," the resulting light image with the characteristics of the distribution of the light field. The work entitled "Lighting engineering and light art" [1] describes two components of success in architecture - creative impulse (talent) and erudition, understanding the laws of light propagation.

However, the modern practice of OU design shows that there is a significant problem for designers without engineering training, which does not fit into training with a pencil, and theories with strict definitions of the quantities used and mathematical relationships between them are needed. Our experience has shown that using computer graphics programs, and you can convincingly present lighting technology on the example of the resulting synthetic images of the designed LI.

We proposed [4] the concept of educating creative abilities in engineering students, where computer graphics programs successfully replaced the famous pencil of designers. In this paper, we conducted classes on the design of LI using the same methodology among engineers and designers. It allowed us to identify the difference in their thinking and formulate principles for overcoming it. This approach can be used as the basis for a new specialty of light design. 


\section{Technique basis}

The basis of our proposed training methodology is the extensive use of computer graphics on the example of professional lighting programs - DIALux and DIALux Evo [5], which allow you to model lighting installations (LI) of any complexity.

In any lighting project, there are always three stakeholders: the customer, the designer, and the designer. Each of them describes and evaluates the quality of the LI in its terminology. Photography (a synthetic image on the screen) creates a common language of communication and understanding. This fact turned out to be crucial, and modeling programs quickly completely replace the classical methods of OU design.

Modern computer lighting programs allow you to consider multiple reflections in the diffuse approximation. In this case, the lighting technician receives not the values of illumination or brightness at individual points of the lighting scene, but the parameters of the light field at all its points. It allows you to represent the distribution of the light field in various forms: tables, isolux curves, fictitious (pseudo) colors, which removes the disadvantages of photorealistic reproduction: the image on the screen is scaled, contrasted, and changed in color. It removes the main problem of LI design: the design object itself - light - unlike any other object, is invisible, and only the created LI will allow you to see all the objects as a whole.

In turn, the image of an object in combination with light distribution analysis tools made it possible to design an OPC not only based on the values of illumination or brightness at fixed points in the scene but also to take into account the quality of lighting: discomfort, light rhythm, accents. By solving the problems of calculating and analyzing the distribution of the light field across the scene and creating an image of the scene, OU modeling programs have opened up broad opportunities for LI design. We are witnessing a new approach to the creation of LI and, accordingly, the emergence of a new profession - lighting design.

Now, classical methods of teaching lighting design are outdated and do not allow students to develop their creative abilities.

Our proposed teaching method lacks the disadvantages of classical teaching methods and has several undoubted advantages:

1. Interaction with the teacher at all critical stages of the project.

2. Training on real projects.

3. Group presentation of projects, discussion, and protection of lighting solutions.

\section{Organization of classes}

thorough preparatory work, and the student - basic knowledge of lighting engineering and work in the DIALux and DIALux Evo lighting programs, which have become the primary tool for any light designer.

The methodology requires careful study of each of the four stages: preparatory and practical stage, presentation, and summing. 
Much attention is paid to the verified timing since the entire process has a limited time frame. The duration of the class is two academic hours (two 45 minutes without a break)

A computer room equipped with a projector and a teacher's computer is required for training. From our experience, the number of jobs and users should not exceed ten people. Before the lesson, pre-prepared models in programs are uploaded to students ' computers. The first 20-30 minutes are the introductory part - the teacher's presentation of the illuminated object. The purpose of the presentation is to introduce the audience to the item and formulate the necessary recommendations and requirements for lighting. For example, if we are talking about Museum lighting, such recommendations are usually: light levels on exhibits, calculating the required level of contrast with the background, choosing lighting devices, choosing the right optics and color temperature, calculating the blinding effect. For each class, you can select particular lighting tasks that students will have to complete.

The preparatory stage includes the following activities:

- the teacher introduces students to the object model in the DIALux/DIALux Evo program;

- demonstration and explanation of the task of working with the model. Both standard tasks and additional tasks of suspended complexity are issued.

Then the practical stage begins - students work on lighting the object in the program. In the course of work, questions to the teacher are welcome. After all, this way, a live dialogue between the teacher and students is achieved. Through virtualization, when an image from any of the students ' computers is displayed on the projector, the teacher can publicly give comments and recommendations. One of the mandatory aspects of high-quality work is to assign a name to each project that reflects the essence of the lighting concept.

Students ' work on lighting modeling continues for 30 minutes, after which the third stage begins - the presentation of projects. Each of the students demonstrates their concept of lighting the object. Simultaneously, all students present in the class participate in the discussion, which opens a broad debate on the proposed solutions in the coverage.

The teacher records each speech, indicates the title and author, and then evaluates it according to the main criteria: visual perception, technical performance, and presentation level. The latter, in our opinion, is essential for students, so in the end, the Foundation is laid in the ability to correctly convey their thoughts and set up communication with the customer.

Then, after individual presentations of students on the protection of their lighting solutions, based on the collected data, the teacher conducts a discussion with students, where the submitted works are discussed. Based on the collected opinions and comments, points are calculated, the best works are selected using the ranking method, and ratings are given.

The same technique can be used to teach artists the "physics of light" - in the created project, and you can clearly show the parameters of light distribution that affect 
the perception of an artistic image: light contrasts, brightness, adaptation, color characteristics, parameters of light sources, and much more.

\section{$4 \quad$ Test sessions}

We conducted an experiment where groups of engineers (the Department of lighting engineering of the NRU "MPEI") and designers (Stroganov Moscow state Academy of art and industry and the Department of computer-aided design and design of the NUST "MISIS") were asked to illuminate the salon of works of art.

Participants were presented and sent models of the Museum salon (see Fig. 1.) in the DIALux / DIALux Evo programs - by choice. The programs are similar in their functionality, and it does not matter in which of the two programs, students will complete the tasks assigned to them. The essence of the task was to illuminate the exhibition objects - paintings and sculptures located in the room, according to the norms and requirements of Museum lighting: the level of illumination on the exhibits, the contrast of objects and background, uneven lighting, brilliance.

The lighting system is based on spotlights installed on a lighting tire track, which is suspended at the height of 4 meters from the floor level and 0.5 meters from walls. Thus, the structure runs along three walls and represents the letter "P" on the plan. a Wide set of lighting devices is already loaded into the Solon model of works of art. Students are invited to choose the correct spotlights in their opinion, place them on the tire track, aim at the illuminated objects And achieve the required lighting indicators.

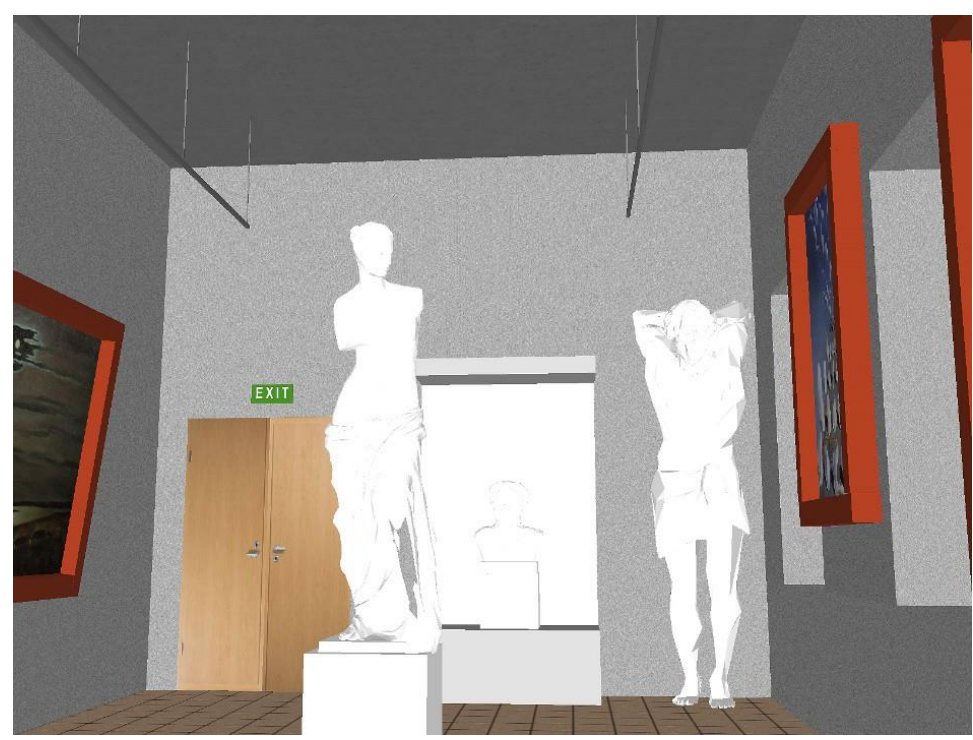

Fig. 1. The original assignment is a three-dimensional view of the Museum interior without lighting. 
This process is complicated by the fact that by lighting any object in the scene with a single lamp, it is not possible to achieve the required indicators. There will always be a need for a combination of floodlights and the need to consider the lighting of the neighboring exhibit. Students are initially forced to choose and use lamps based on the description of their technical characteristics, perform calculations in the program and adjust the choice of lighting equipment based on the results of the calculation analysis. In our opinion, this iterative method of selection and subsequent refinement opens up opportunities for students to show their creative abilities. The result of the task is a generated lighting report, which the program creates from the calculated data that the listener submits for protection to prove the correct implementation of lighting in the cabin.

From the results of the work of Engineers (Fig. 2) and designers (Fig. 3), we can conclude that all students, without exception, understood the task and demonstrated a reasonably high level of knowledge of the programs.

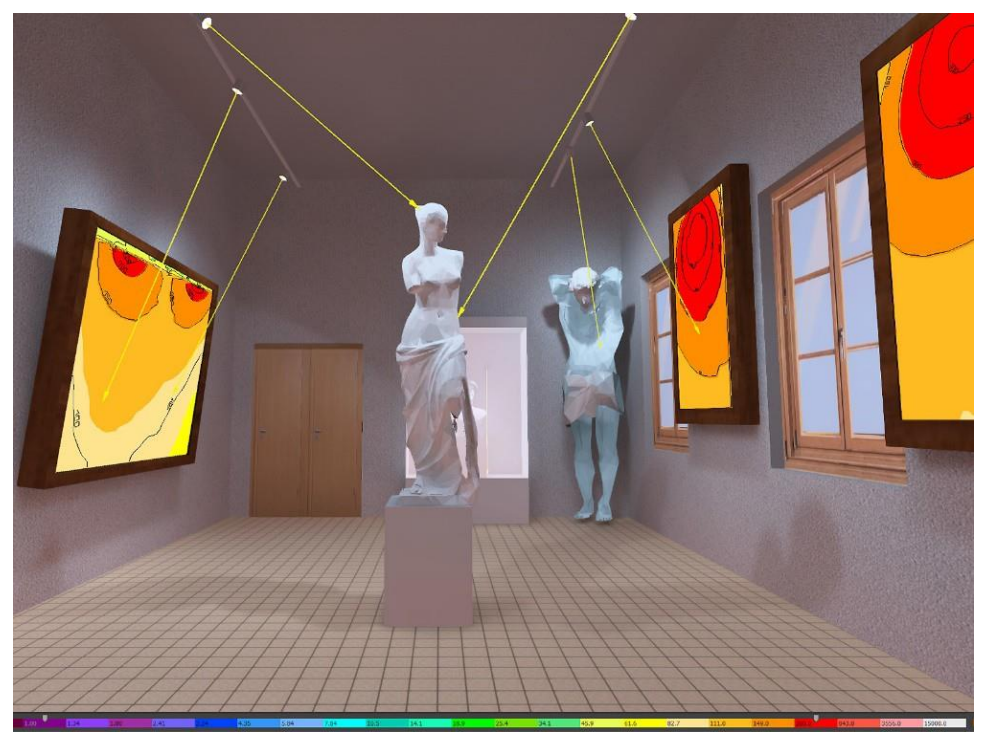

Fig. 2. The engineering approach in lighting (MPEI). Searchlights are arranged according to the task. The targeting of lighting devices and the light levels on the exhibits are shown.

I would especially like to note the difference in approaches to solving lighting problems of engineers and designers. Engineers used a limited set of spotlights and tried to reduce their number to a minimum, used the method of lowering and increasing the luminous flux from the lamps, and followed the instructions precisely to arrange the lighting equipment. Designers, on the other hand, added and used lamps in places not specified in the task, and paid more attention to the light and color environment of the room than to strict achievement of light levels and unevenness. 


\section{Conclusions}

Our proposed method of teaching students, based on computer programs for modeling lighting and its testing by engineers and designers, showed different approaches in the thinking of students when implementing lighting. In the process of public defense of their concepts and the presence of qualified teachers, it became possible to visually correct approaches to lighting and rethink the possibilities of light design.

Thus, we are convinced that the further development of this technique, based on the use of computer graphics programs, will lay the foundation for creating a singlespecialty - light design.

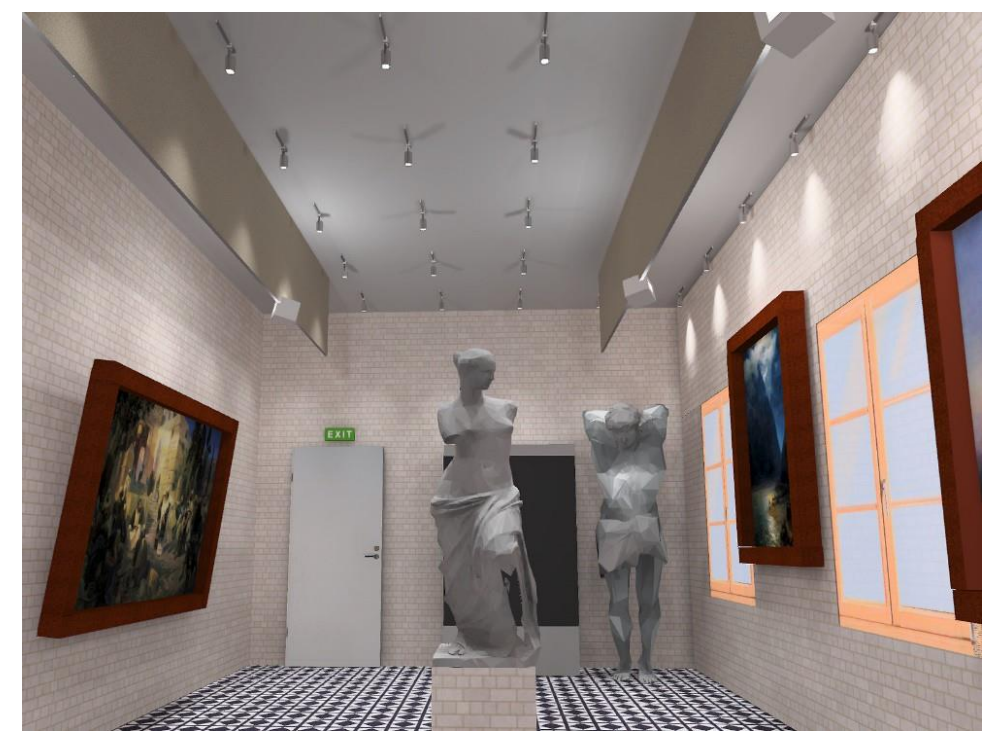

Fig. 3. The artistic approach to lighting (MISIS). Spotlights are positioned almost evenly and independently on the ceiling. Added ceiling lighting fixtures to expand the space.

\section{References}

1. Makarevich, V.G.: Lighting engineering and light art. Svetotekhnika, 10, $15-17$ (1974)

2. Matveev, A.B., Tokhadze, I.L., Undasynov, G.N.: Similarity criterion for reproducing brightness ratios. Svetotekhnika, 11, 5-8 (1968)

3. Matveev, A.B.: Methods of modeling lighting installations. Svetotekhnika, 10, 1-2 (1971)

4. Budak, V.P., Kovyrkova, M.D., Makarov, D.N., Minaeva, S.Yu., Skornyakova, A.A.: Light design: the education of creative abilities in lighting engineering students. Svetotekhnika, 1, 80-83 (2019)

5. DIAL, https://www.dial.de, last accessed 2016/09/05 